\title{
Dynamic Growth Structures in Diamonds from Bingara, NSW, Australia
}

\author{
Davies, R. M. ${ }^{1}$, O'Reilly, S. Y. ${ }^{1}$, Griffin, W. L. ${ }^{1,2}$
}

1. GEMOC National Key Centre, Macquarie University, Sydney, NSW, 2109, Australia

2. CSIRO Exploration and Mining, P. O. Box 136, North Ryde, NSW, 2113, Australia

The internal structure of diamonds can be qualitatively studied using electron microscope or cathodoluminescence (CL) imagery. CL highlights variations in impurity distribution, and CL images can show changes in morphology during growth, dissolution and deformation, and may provide a spatial context for interpretation of nitrogen content and nitrogen aggregation states measured by infrared spectroscopy (eg. Bulanova, 1995). CL data are usually collected as colour images. Colour variations on a CL image are mainly related to variations in nitrogen content between different parts of the diamond, and to a lesser extent to the presence of other trace impurities. Nitrogen is the most common impurity incorporated in the diamond lattice (Type I) and produces a variable blue colour on the CL image. Yellow CL colours are less common, and do not appear to indicate incorporated impurities. Rather, they occur in stress or defect zones with low nitrogen contents, such as amorphous seeds (commonly hydrogen-bearing), around mineral inclusions and radiation spots, and along deformation laminae. Regions with trace amounts of nitrogen have dark CL colours.

Alluvial diamonds from Bingara and Wellington in eastern Australia are dominated by a unique population of diamonds, herein termed Group B: these have highly resorbed crystal forms, distinctive surface features, calcium-rich eclogitic inclusions accompanied by abundant coesite inclusions, and heavy carbon isotope signatures (J13C $-5 \%$ o to $+3 \%$; see summary by Davies et al., this volume). Like other eastern Australian alluvial diamonds, the Group B diamonds come from an unidentified source and occur in a Phanerozoic tectonic setting. CL studies of these diamonds have been carried out as part of a larger study which aims to characterise the diamonds, understand their origin, and constrain exploration models.

\section{Results}

Polished central plates and faces of 54 Group B diamonds were investigated using CL. The diamonds were divided into two groups, based on surface morphology, internal CL, and infrared absorption characteristics: a yellow group with high nitrogen contents and nitrogen aggregation states (1000 $2500 \mathrm{ppm} ; 18$ to $42 \% \mathrm{IaB})$, and the presence of carbonate and hydrogen, and a white and brown group with low to trace amounts of nitrogen $(<400 \mathrm{ppm} \mathrm{N}$ and $<15 \% \mathrm{IaB}$ to $1000 \mathrm{ppm}$ and $60 \% \mathrm{IaB}$ in some cores). All Group B diamonds have lustrous low-relief resorbed surfaces without primary octahedral faces. Common surface features include deformation laminae which are only partially developed and cross-cut the stones in several directions. The deformation laminae occur as raised features that often show herringbone structure. This is not a twinning feature but represents lessresorbed surfaces of the stone that retain evidence of growth layering while the surrounding surfaces are more rounded by resorption. The low-nitrogen diamonds show greater evidence of deformation, containing small strain hillocks, lamination lines and deep cavities on the resorbed surfaces.

CL images of the nitrogen-rich group of diamonds are homogeneous and blue. A few of these diamonds show planar internal structures, which indicate octahedral form and growth in stable conditions (Bulanova, 1995). Examples of this occur in which Type II intergrowths and rims are aligned with octahedral planes. In contrast, diamonds of the nitrogen-poor group have complex internal structures, with thick concentric non-planar Type Ia and Type II layers that may indicate unstable and dynamic growth histories, during which deformation occurred simultaneously with diamond growth. More than half of the low-nitrogen Group B stones show a large rounded nitrogen rich (ca $900 \mathrm{ppm})$ central structure, possibly representing a resorbed form. The round centres are overgrown by nitrogen-poor diamond which is either continuous out to the rim of the stone, or may be overgrown by an oscillating sequence of Type Ia and Type II layers with sharp contacts, suggesting growth under rapidly changing conditions. Within the round central structures, small Type II triangular shards may occur, indicative of the interplay of (111) octahedral planes integrating Type 
II cube (100) facets on a micro-scale (Frank et al., 1990). On a macro-scale only non-planar facets are observed, and unlike diamond from other occurrences (eg. Group A diamonds from Wellington, NSW; Davies et al., this volume), planar octahedral layers never assimilate the less stable rounded facets as growth proceeds (Figure 1).

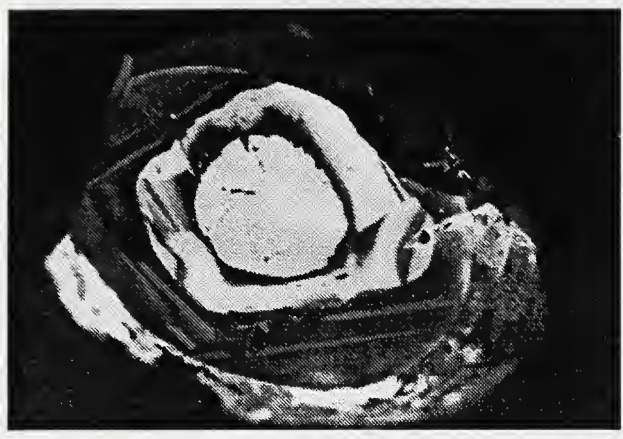

Figure 1. CL image of non-planar concentric zoning with a nitrogen -rich centre.

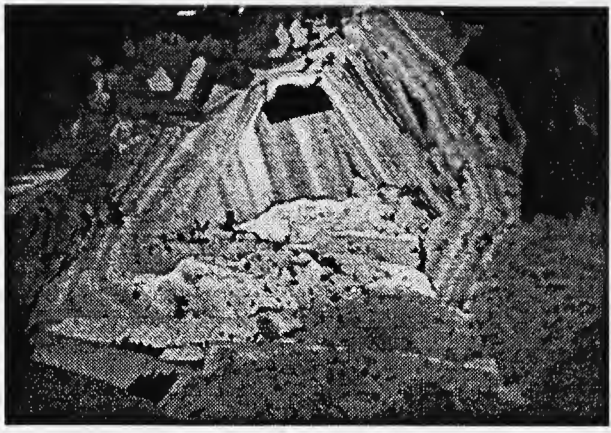

Figure 2. Reniform structures outside central zone.

The non-planar concentric zoning structures are comparable to those seen in zoned grandite garnets from hydrothermal systems (Jamtveit and Andersen, 1994). Other non-planar structures, occurring in about a third of these diamonds, include sub-parallel bands with thick brush-stroke textures that may be a form of sector structure. One diamond shows reniform structures (Figure 2); in another the central zone contains dendritic structures and part of the rim zone contains a cluster of small oscillatorily-zoned cube forms that appear to be growth nuclei (Figure 3).

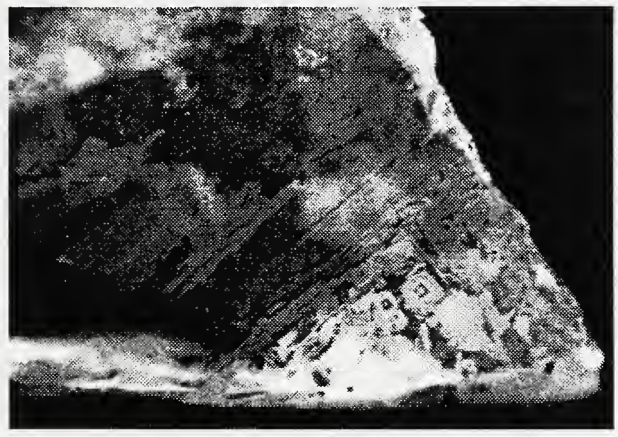

Figures 3. Lobed structures with a cluster of small oscillatorily zoned cube forms on rim (bottom right).

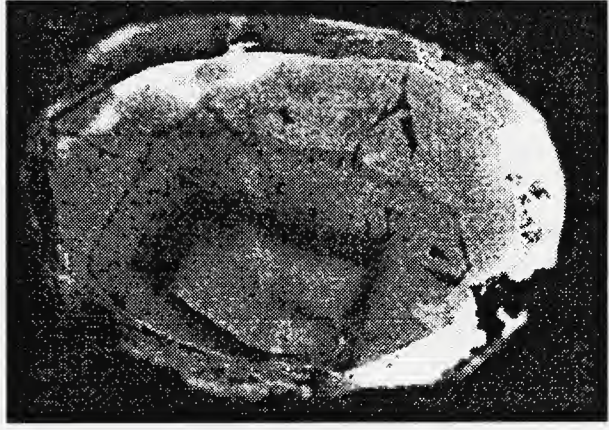

Figure 4. Brittle deformation structures showing displacements of zoning with Type II annealing.

Deformation is ubiquitous in the low-nitrogen Group B diamonds. However, it is evident not as the fine cross-hatched linear features typical of most plastic deformation in diamond, but as brittle displacements of layers throughout the stones (Fig. 4). Stronger deformation produces cataclastic disruption of layers and brecciation of internal structures (Figure 5). In an extreme case, the diamond consists of small crystallites with a mottled blue texture in CL, apparently deformed to the point of recrystallisation. Where strain displacements occur, Type II diamond anneals the ruptured zones. Deformation zones may be overgrown by undeformed diamond, showing that diamond growth was accompanied by and also post-dated deformation. 


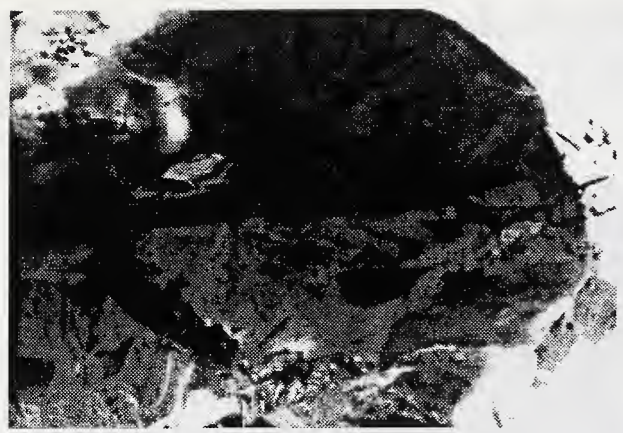

Figure 5. Cataclastic deformation structures with Type I and Type II diamond.

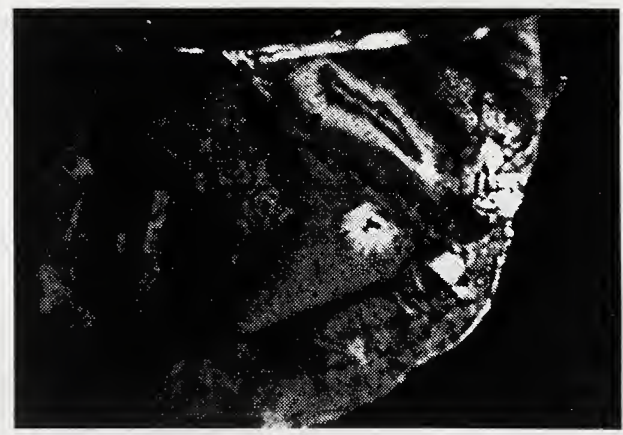

Figure 6. Diffuse N-rich domains in N-poor diamond.

Deformation in zones of diamonds with trace amounts of nitrogen appears as a network of fine lines of blue CL on a dark background. Nitrogen has apparently diffused along these cracks, sometimes concentrating as diffuse domains of blue CL independent of growth zoning (Figure 6). This feature may provide important insights into nitrogen aggregation in diamond, as it is evidence that deformation (Evans, 1992), as well as temperature and time (Allan and Evans, 1981) promotes the aggregation process. The high-nitrogen diamonds show little evidence of deformation, apart from the linear features on the diamond surfaces. Apparently CL is unable to identify evidence of deformation in these diamonds, possibly due to the high nitrogen content. Alternatively, it is possible that high nitrogen contents may somewhat inhibit deformation (Milledge pers. comm., 1996).

\section{Conclusion}

The Group B diamonds may have grown rapidly in a stress field. A group of nitrogen-poor diamonds shows complex structures comparable to those in minerals grown in hydrothermal systems. The inability of these diamonds to attain ideal octahedral or cubic forms throughout their growth histories, combined with displacements of growth zones and the occurrence of dendritic structures and other non-planar growth forms, suggests that they grew rapidly during and following episodes of high strain. In the same population, a nitrogen-rich group of stones containing traces of carbonate and hydrogen have homogenous blue CL structures. These diamonds may have also grown rapidly, incorporating abundant impurites (Navon et al., 1988). The nitrogen-poor diamond group may have formed at lower oxygen fugacities (Humbert et al., 1997), at higher temperatures and hence higher rates of lattice diffusion (Watson, 1997), or in a more nitrogen-depleted environment, than the highnitrogen diamonds.

\section{References}

Allen, B. P. and Evans, T., 1981, Aggregation of nitrogen in diamond, including platelet formation: Proc. Roy. Soc. Lond., A375, p. 93-104.

Bulanova, G. P., 1995, The formation of diamond: J. Geochem. Expl., 53, p. 1-23.

Evans, T., 1992, Aggregation of nitrogen in diamonds: In: The Properties of Synthetic and Natural Diamonds (J. E. Field, ed.). Academic Press, p. 259-290.

Frank, F. C., Lang, A. R., Evans, D. J. F., Rooney, M. L. T., Spear, P. M. and Welbourn, C. M., 1990, Orientationdependent nitrogen incorporation on vicinals on synthetic diamond cube growth surfaces: J. Cryst. Growth, 100, p. 354-376.

Humbert, F., Libourel, G., Marty, B., France-Lanord, C., 1997, Nitrogen solubility in silicate melt under oxidized and reduced conditions using laser extraction/static mass spectrometry analysis: Abstr. Seventh Ann. V. M. Goldschmidt Conf., p. 101-102.

Jamtveit, B. and Andersen, T.B. 1994. Morphological instabilities during rapid growth of metamorphic garnets. Phys. Chem. Min., 19, 176-184.

Navon, O. Hutcheon, I. D., Rossman, G. R., and Wasserburg, G. J., 1988., Mantle-derived fluids in diaond microinclusions: Nature, 335, p. 784-789.

Watson, E. B., 1996, Surface enrichment and trace-element uptake during crystal growth: Geochim. Cosmochim. Acta, 60 , p. 5013-5032. 